\title{
Preictal and Ictal Neurovascular and Metabolic Coupling Surrounding a Seizure Focus
}

\author{
Mingrui Zhao, ${ }^{1}$ John Nguyen, ${ }^{2}$ Hongtao Ma, ${ }^{1}$ Nozomi Nishimura, ${ }^{2}$ Chris B. Schaffer, ${ }^{2}$ and Theodore H. Schwartz ${ }^{1}$ \\ ${ }^{1}$ Department of Neurological Surgery, Weill Medical College of Cornell University, New York Presbyterian Hospital, New York, New York 10021, and \\ 2Department of Biomedical Engineering, Cornell University, Ithaca, New York 14853
}

Epileptic events initiate a large focal increase in metabolism and cerebral blood flow $(\mathrm{CBF})$ to the ictal focus. In contrast, decreases in CBF have been demonstrated surrounding the focus, the etiology of which is unknown (i.e., arising either from active shunting of blood or passive steal). The relationship between these events and neuronal activity and metabolism are also unknown. We investigated neurovascular and neurometabolic coupling in the ictal surround using optical imaging of light scattering and cerebral blood volume, autofluorescence flavoprotein imaging (AFI), direct measurements of the cortical metabolic rate of oxygen and two-photon imaging of blood vessel diameter in a rat model of ictal events elicited with focal injection of 4-aminopyridine. We discovered a novel phenomenon, in which ictal events are preceded by preictal vasoconstriction of blood vessels in the surround, occurring $1-5 \mathrm{~s}$ before seizure onset, which may serve to actively shunt oxygenated blood to the imminently hypermetabolic focus or may be due to small local decreases in metabolism in the surround. Early ictal hypometabolism, transient decreases in cell swelling and cerebral blood volume in the surround are consistent with early ictal surround inhibition as a precipitating event in seizure onset as well as shaping the evolving propagating ictal wavefront, although the exact mechanism of these cerebrovascular and metabolic changes is currently unknown. AFI was extremely sensitive to the ictal onset zone and may be a useful mapping technique with clinical applications.

\section{Introduction}

Neurovascular and neurometabolic coupling are critical to supply the energy demands of brain tissue during both normal physiological function and pathological conditions such as epilepsy (Raichle and Mintun, 2006; Iadecola and Nedergaard, 2007). Early investigators have shown dramatic increases in cerebral blood flow (CBF) and metabolism in the epileptic focus during seizure initiation and propagation in a variety of animal models, originally using techniques such as autoradiography and more recently in humans with PET, SPECT, fMRI, and intrinsic optical signal (IOS) imaging (Folbergrová et al., 1981; Ingvar, 1986; Kreisman et al., 1991; Kobayashi et al., 2006; Zhao et al., 2007; RocheLabarbe et al., 2008). Preictal hemodynamic events, which may precede seizures by anywhere from $20 \mathrm{~min}$ to a few seconds, have also been described, showing promise as a technique for identifying seizure onsets (Diehl et al., 1998; Zhao et al., 2007). However, little attention has been paid to neurovascular and neurometabolic coupling and preictal activity in the area of cortex surrounding the epileptic focus during seizures. The "sur-

\footnotetext{
Received May 25, 2011; revised July 8, 2011; accepted July 14, 2011.

Author contributions: M.Z., J.N., C.B.S., and T.H.S. designed research; M.Z., J.N., H.M., and N.N. performed research; M.Z. and J.N. analyzed data; M.Z., J.N., C.B.S., and T.H.S. wrote the paper.

This work was supported by NINDS Grant R01 NS49482 (T.H.S.), the American Society for Laser Medicine and Surgery, the Johnson \& Johnson Cornell Seed Grant, a grant from the Barraket and Davis Foundations (T.H.S., C.B.S.), the NSF GK-12 program (J.N.), and Clinical and Translational Science Center (CTSC) Grant UL1 RR 024996 (C.B.S., M.Z.). We thank Drs. A. Gjedde and K. J. Thomsen for help with $\mathrm{CMRO}_{2}$ measurements.

Correspondence should be addressed to either Dr. Mingrui Zhao or Dr. Theodore H. Schwartz, Department of Neurological Surgery, Weill Medical College of Cornell University, 525 East 68th Street, Box 99, New York, NY 10065. E-mail:miz2003@med.cornell.edu or schwarh@med.cornell.edu.

DOI:10.1523/JNEUROSCI.2597-11.2011

Copyright $\odot 2011$ the authors $\quad 0270-6474 / 11 / 3113292-09 \$ 15.00 / 0$
}

round" of an epileptic focus was originally identified in interictal animal models of epilepsy, using penicillin injection or other $\mathrm{GABA}_{\mathrm{A}}$ antagonists, as an area of intense neuronal inhibition, presumed to prevent the transition to ictal onset (Prince and Wilder, 1967). This so called "inhibitory surround," however, was not commonly found during ictal events, except in in vitro models where feed-forward inhibition was shown to act as a "veto" mechanism to limit seizure spread (Trevelyan et al., 2007).

Hemodynamic imaging techniques also show profound inverted signals in the cortex surrounding an ictal event, indicating decreases in CBF and cerebral blood volume (CBV) (Schwartz and Bonhoeffer, 2001; Zhao et al., 2009). Studies of cortical metabolism using tools such as PET or autoradiography, however, do not routinely show hypometabolism in the cortex surrounding the seizure focus (Engel et al., 1982). In our previous studies of acute seizures, we have demonstrated a transient decrease in $\mathrm{CBF}$ and $\mathrm{CBV}$ around an ictal focus, accompanied by an increase in tissue oxygenation (Zhao et al., 2009). The etiology of this short drop in perfusion was unclear. In this current study, we examined whether the decrease in $\mathrm{CBF}$ and $\mathrm{CBV}$ around a seizure focus was accompanied by a decrease in cortical metabolism. We also examined whether the decrease in $\mathrm{CBF}$ and $\mathrm{CBV}$ is caused by a passive shunting of blood into the ictal focus (i.e., a steal phenomenon caused by vasodilation in the focus) or by active shunting of blood due to vasoconstriction in the surrounding brain. Finally, we studied the timing of these processes with the goal of identifying preictal neurovascular or neurometabolic events in the surround that may elucidate mechanisms for seizure initiation. We found that metabolism is decreased in the cortex surrounding a seizure focus and discovered a new phenomenon, 
namely, preictal vasoconstriction in a ring of cortex surrounding the seizure focus, which actively shunts blood to the ictal focus and may serve to prepare the focus for the impending dramatic increase in neuronal activity and metabolism. The significance and possibly etiologies of this new finding are discussed.

\section{Materials and Methods}

Animal preparation. All experimental procedures were approved either by the Weill Cornell Medical College or Cornell University Animal Care and Use Committee following NIH guidelines. Adult male Sprague Dawley rats (250-380 g) were anesthetized and maintained stable at normal values, as previously described (Zhao et al., 2009). Animals were placed in a stereotaxic frame. An $\sim 5 \times 8 \mathrm{~mm}$ cranial window was opened over one hemisphere to expose somatosensory cortex and surrounding brain. The dura was carefully removed. To visualize the surface vasculature in the two-photon excited fluorescence imaging experiments, $\sim 0.3 \mathrm{ml}$ of $5 \%$ (wt/vol) $2 \mathrm{MDa}$ fluorescein-conjugated dextran (FD2000S; Sigma) in saline was intravenously injected through the tail vein.

Epileptogenesis and electrophysiology. Ictal discharges were induced by injecting the potassium channel blocker 4-aminopyridine (4-AP; Sigma; $15 \mathrm{~mm}, 0.5 \mu \mathrm{l})$ through a single-barreled glass microelectrode using a Nanoject II injector (Drummond Scientific) (Schwartz and Bonhoeffer, 2001; Rensing et al., 2005; Motamedi et al., 2006; Xu et al., 2008; Zhao et al., 2009). Extracellular local field potential (LFP) was recorded either with the same electrode or a second single-barreled glass microelectrode (impedance, 2-4 M $\Omega$ ) filled with $0.9 \%$ saline, positioned $<1 \mathrm{~mm}$ from the 4-AP electrode, and lowered to a depth of 300 500 $\mu \mathrm{m}$ into the neocortex. The signal was amplified and filtered between 0.1 and $500 \mathrm{~Hz}$ using a DAB-S system (World Precision Instruments), and digitized at $1000 \mathrm{~Hz}$ by a CED Power 1401 (Cambridge Electronic Design). Data were recorded by a PC running the Spike2 software (Cambridge Electronic Design).

Laser Doppler flowmetry. One custom-angled stainless steel laser Doppler flowmetry (LDF) probe was placed on the cortex, avoiding large blood vessels (wavelength, $780 \mathrm{~nm}$; fiber separation, $0.25 \mu \mathrm{m}$; Perimed). The probe was placed as close as possible to the 4-AP electrode (hereafter referred to as the "focus") or $>2 \mathrm{~mm}$ away from the electrode (hereafter referred to as the "surround"). Nine rats and eight rats were used in the focus experiment and the surround experiment, respectively. LDF provides continuous hemodynamic monitoring of red blood cell speed and red cell concentration, yielding a calculated CBF measure that correlates with traditional methods (Skarphedinsson et al., 1988). CBF was continuously recorded by the PeriFlux System 5000 (Perimed). Data were acquired at $200 \mathrm{~Hz}$ with a $0.05 \mathrm{~s}$ time constant using a CED Power 1401 and Spike2 software.

Tissue oxygenation. Tissue oxygenation was measured with one Clarkstyle polarographic oxygen microsensor, which responds linearly to tissue oxygen concentration changes. The tip of the microsensor is $25 \mu \mathrm{m}$ in diameter with a $90 \%$ response time of $<0.5 \mathrm{~s}$. The tip measures a sphere of tissue $\sim 60 \mu \mathrm{m}$ in diameter. Calibrations were performed at $37^{\circ} \mathrm{C}$ in saline equilibrated with either bubbling air [atmospheric partial pressure of oxygen $\left(\mathrm{pO}_{2}\right)$ ] or $100 \% \mathrm{~N}_{2}$ gas $\left(0 \mathrm{pO}_{2}\right)$ before each experiment. The oxygen microelectrode was inserted $\sim 400 \mu \mathrm{m}$ deep into the neocortex. It was located as close as possible to the 4-AP injection site (focus) or $>2$ $\mathrm{mm}$ away (surround). In both cases, the oxygen microsensor was placed as close as possible to the LDF probe. The signal was measured with a high-impedance picoammeter (PA 2000; Unisense A/S) and recorded to the computer by a CED Power 1401 and Spike2 software. An off-line 0.3 $\mathrm{Hz}$ low-pass filter was used to remove the artifacts caused by spatial displacement (Masamoto et al., 2003; Offenhauser et al., 2005).

Oxygen consumption. Cortical metabolic rate of oxygen $\left(\mathrm{CMRO}_{2}\right)$ was calculated from $\mathrm{CBF}$ and $\mathrm{pO}_{2}$ measurements as described by Gjedde (2006) and Thomsen et al. (2009). The relationship among the three variables is as follows:

$$
t P_{\mathrm{O}_{2}}=P_{50} \sqrt[h]{\frac{2 C_{a} C B F}{C M R O_{2}}-1}-\frac{C M R O_{2}}{2 L},
$$

where $t P_{\mathrm{O} 2}$ is the tissue oxygen tension, $P_{50}$ is the half-saturation tension of the oxygen-hemoglobin dissociation curve, $h$ is the Hill coefficient of the same dissociation curve, $C_{a}$ is the arterial oxygen concentration, and $L$ is the effective diffusion coefficient of oxygen in brain tissue. The value of $L$ was determined from baseline values of rats in similar conditions of anesthesia in which $\mathrm{CBF}$ and $\mathrm{CMRO}_{2}$ were reported in the literature to be $53 \mathrm{ml} \cdot 100 \mathrm{~g}^{-1} \cdot \mathrm{min}^{-1}$ and $219 \mu \mathrm{mol} \cdot 100 \mathrm{~g}^{-1} \cdot \mathrm{min}^{-1}$, respectively (Zhu et al., 2002). The corresponding value of $L$ was $5.45 \mu \mathrm{mol} \cdot 100$ $\mathrm{g}^{-1} \cdot \mathrm{min}^{-1} \cdot \mathrm{mmHg}^{-1}$ for standard values of $P_{50}(36 \mathrm{mmHg}), h(2.7)$, and $C_{a}\left(8 \mu \mathrm{mol} \mathrm{ml}^{-1}\right)$.

Intrinsic optical imaging. The exposed cortical surface was illuminated with one of two different wavelengths $(570 \pm 10$ and $810 \pm 30 \mathrm{~nm})$ of light. Reflected light from the cortical surface was collected by a $50 \mathrm{~mm}$ camera lens and divided into two separate paths by a dichroic beamsplitter. Each light beam passed through a second $50 \mathrm{~mm}$ camera lens. The reflectance at $570 \mathrm{~nm}$ wavelength provides a measurement of CBV. At $800 \mathrm{~nm}$, the optical signal is largely derived from changes of light scattering related to cell swelling as well as intracellular and extracellular fluid shifts, which provide an indirect representation of neuronal activity (Salzberg et al., 1985). Images were acquired at $10 \mathrm{~Hz}$ (Imager 3001; Optical Imaging) as previously described (Zhao et al., 2009). Intrinsic imaging was performed in five rats at each wavelength by manually switching the filters between trials.

Autofluorescence flavoprotein imaging. AFI is a nonhemodynamic measure of cellular metabolism derived from the fluorescence of flavoproteins associated with the electron transport chain in mitochondria. We imaged flavoprotein autofluorescence using a bandpass excitation filter $(455 \pm 35 \mathrm{~nm})$, an extended reflectance dichroic mirror $(500 \mathrm{~nm})$, and a $>515 \mathrm{~nm}$ long-pass emission filter (Reinert et al., 2007), on the same imaging system described above. AFI was performed on the same five rats simultaneously with the $810 \mathrm{~nm}$ data since there was no overlap between the wavelengths.

Two-photon microscopy of surface cortical vasculature. In vivo images of cortical vasculature were obtained with a custom-built two-photon excitation fluorescence microscope using low-energy, $100 \mathrm{fs}, 800 \mathrm{~nm}, 76$ $\mathrm{MHz}$ repetition rate laser pulses produced by a titanium:sapphire oscillator (Mira-HP; Coherent) that was pumped by a continuous wave laser (Verdi-V18; Coherent). Laser scanning and data acquisition were controlled using MPSCOPE software (Nguyen et al., 2006). To help navigate and map the entire cranial window, we used a 0.28 numerical aperture (NA), $4 \times$ magnification, air objective (Olympus). For high-resolution imaging and vessel diameter measurements, we used a $0.95 \mathrm{NA}, 20 \times$ magnification, water-immersion objective (Olympus). High-resolution movies (3.39 frames per second) of vessels were obtained to track diameter changes during seizure activity. Four rats were used in the vessel diameter measurement.

Data analysis. The offline analysis was performed using custom analysis software written in Matlab (MathWorks). Since ictal events can have a varying electrographic morphology, we only analyzed those that had no interictal spikes during the $20 \mathrm{~s}$ before the ictal onset and that began with a large population spike followed by a recruiting rhythm (typical seizure) to eliminate any confusion regarding the onset of the seizure (see Fig. 1). Seizure onset was determined by visual analysis of the LFP from the initial negative deflection of the large spike that preceded all seizures (Zhao et al., 2009). $\mathrm{CMRO}_{2}$ data were converted to percentage change $(\Delta)$ from baseline by subtracting then dividing the average baseline value obtained over a $2 \mathrm{~s}$ block of time beginning $2 \mathrm{~s}$ before the onset of the ictal discharge. To look for preictal activity, the $2 \mathrm{~s}$ block was chosen starting $20 \mathrm{~s}$ before seizure onset. The magnitude of the response was estimated as the area under the curve above (positive) or below (negative) two SDs from the pre-event baseline.

Reflectance changes at 800 and $570 \mathrm{~nm}$ were expressed as the percentage of a negative change from the baseline $-\Delta R / R$, while flavoprotein autofluorescence is expressed as the percentage of fluorescence change from the baseline $\Delta F / F$. Response time courses were extracted from 0.5 $\mathrm{mm}$ concentric rings centered on the focus of the 4-AP seizure. We used concentric rings at a predetermined distance to avoid selection bias resulting from placing a region of interest (ROI) by hand (see Fig. 1A). 
Each frame following the onset of each seizure and for $20 \mathrm{~s}$ before onset, was divided by an average of the 20 frames (2s) before the onset. To look for preictal activity, the $2 \mathrm{~s}$ block was chosen staring $20 \mathrm{~s}$ before seizure onset. The amplitude of the response was shown as positive or negative based on whether the signal rose or decreased either above or below a threshold set at two SDs from the pre-event $2 \mathrm{~s}$ baseline. An average of percentage fractional changes of optical signal, positive peak, and negative peak at each time point of optical signal inside each of $0.5 \mathrm{~mm}$ ring were used for further analysis.

For analysis of two-photon image data, Matlab code was developed to calculate diameter changes in individual blood vessels. For each vessel, an ROI was placed over the vessel and its boundary determined using a threshold (20\% of maximum intensity). The diameter was then calculated as the average width across the length of the selected region. A trend line of diameter changes was calculated at baseline and during seizure activity. To determine the measured vessel eliciting a significant response, we calculated the $99 \%$ confidence interval about the mean of baseline activity before seizure onset. We used the upper and lower bounds of the confidence interval as threshold values for dilation and constriction, respectively. The dilation or constriction onset is the time point at which the diameter trend line exceeds the threshold values (see Fig. $4 A$ ). If the trend line does not exceed the bound limit, then the vessel was deemed to not elicit the respective vascular response to the seizure activity. As described above, seizure onset was determined by a distinct spike in the LFP recording. For boxplots, circles indicate individual data points, while cross-hairs are considered statistical outliers, which were not included when calculating the mean. Horizontal red and black lines represent the mean and median, respectively.

For all data, statistical significance was determined with ANOVA and post hoc tests comparing the baseline mean measurement of results averaged over all seizures in all animals. All data were expressed as means \pm SEM.

\section{Results}

\section{Acute focal 4-AP seizures}

Acute focal seizures were elicited with injection of 4-AP. These seizures typically began with a large negative spike followed by a low-amplitude fast activity, which evolved into rapid spike-and-wave activity that gradually increased in periodicity and decreased in amplitude until the seizure terminated (Fig. $1 B$ ).

\section{Light scattering and CBV imaging of center-surround dynamics}

Optical recording at $800 \mathrm{~nm}$ demonstrated an antagonistic center-surround dynamic (Fig. $1 C$ ). The complex, dynamic relationship between the positive signal in the focus and the negative signal in the surround evolved over the course of the seizure. For the purposes of data analysis, we chose two moments in time that were representative of the predominant interaction, namely, the time point at which the positive signal in the seizure focus was at its maximum, an average of $36.5 \pm 8.6 \mathrm{~s}$ after seizure initiation and the time point at which the negative signal in the seizure surround was at its maximum, an average of $11.6 \pm 9.3 \mathrm{~s}$ after seizure initiation. Average data over all experiments revealed that
B
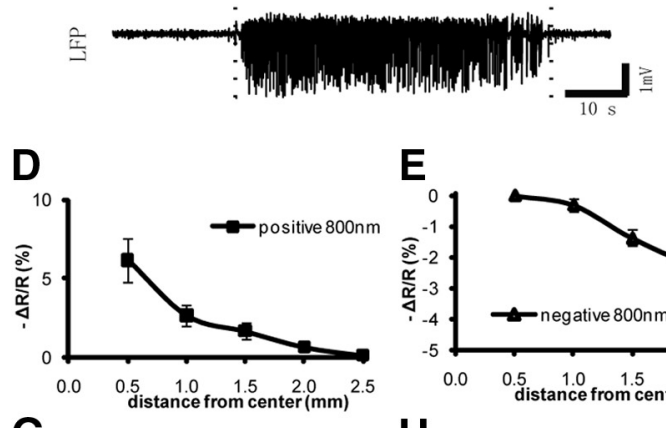

E

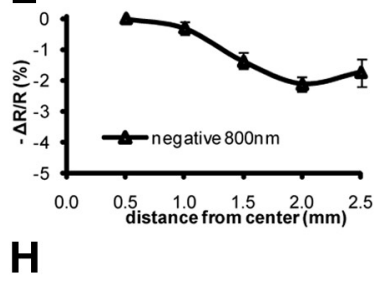

c

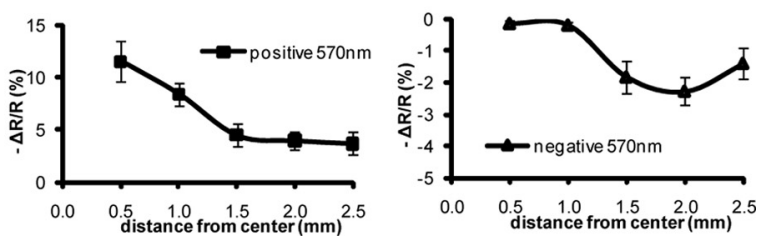

Figure 1. Optical measurements of light scatter and CBV during ictal discharge demonstrate a negative signal in the surround. 列 CBV ( $n=5$ rats, 18 seizures) at its peak is maximal in the focus and falls off with distance. $\boldsymbol{H}$, Average decrease in (BV at the time of its minimum occurs at $\sim 2 \mathrm{~mm}$ from the ictal focus. Error bar, SEM.

the positive signal diminished in amplitude with increasing distance from the seizure focus (Fig. $1 D)$ ( $n=5$ rats, 11 seizures). The maximum amplitude of the positive signal in the focus was $6.2 \pm 1.3 \%$. Statistical testing revealed a significant decline in the positive signal within each ring to a value of $0.14 \pm 1.0 \%$ in the last ring at $2.5 \mathrm{~mm}$ (ANOVA, $p<0.001$ ). The inverted, negative signal in the surrounding cortex, on the other hand, was largest at a distance of $2.0 \mathrm{~mm}$ from the focus with a maximal amplitude of $-2.1 \pm 0.2 \%$, which was also statistically different from the values in other rings (ANOVA, $p<0.001$ ) (Fig. $1 E$ ). Although not a direct measure of neuronal activity, the signal indirectly represents increases in neuronal activity in the focus and decreases in neuronal activity in the surround by measuring mostly changes in light scattering in the tissue (see Materials and Methods).

Optical recording at $570 \mathrm{~nm}$ provided a map of CBV during seizure activity (Fig. $1 F$ ), nearly identical to those previously published by our group (Zhao et al., 2009). The $570 \mathrm{~nm}$ map revealed a similar center-surround effect as seen with the $800 \mathrm{~nm}$ data, but the activity was less focal and had more vascular artifact, which was not seen in the $800 \mathrm{~nm}$ data (Fig. $1 F$ ). The maximal increase in CBV in the focus was $11.5 \pm 1.9 \%$, which decreased with distance but did not reach baseline, even $2.5 \mathrm{~mm}$ from the focus (ANOVA, $p<0.001$; Fig. $1 G, n=5$ rats, 18 seizures). A decrease in $\mathrm{CBV}$ in the surround was also identified with a maximum change of $-2.3 \pm 0.4 \%$ peaking at $2.0 \mathrm{~mm}$ from the focus (ANOVA, $p<0.01$; Fig. $1 H$ ). Hence, CBV maps provide an indirect and less spatially precise measurement of excitatory and inhibitory pattern of neuronal activity, as measured by changes in blood volume, compared with those measured with light scatter changes (Fig. 1C-E), and these maps clearly show a decrease in CBV surrounding a seizure focus. The etiology of this negative 

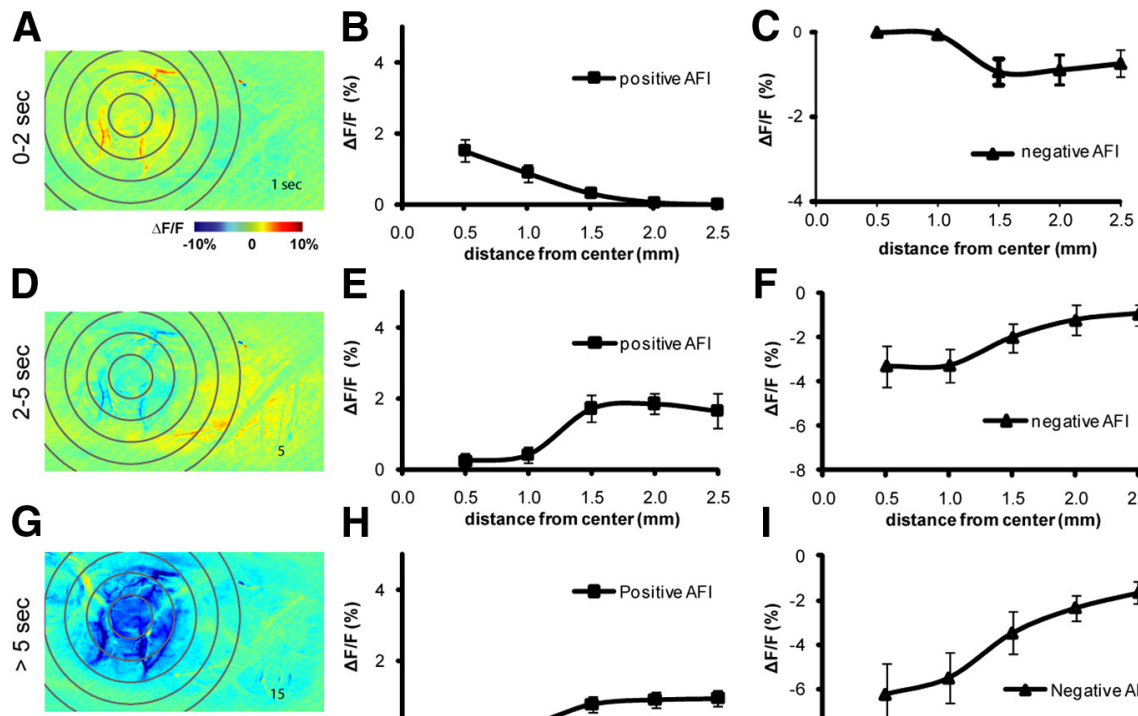

$\mathrm{H}$
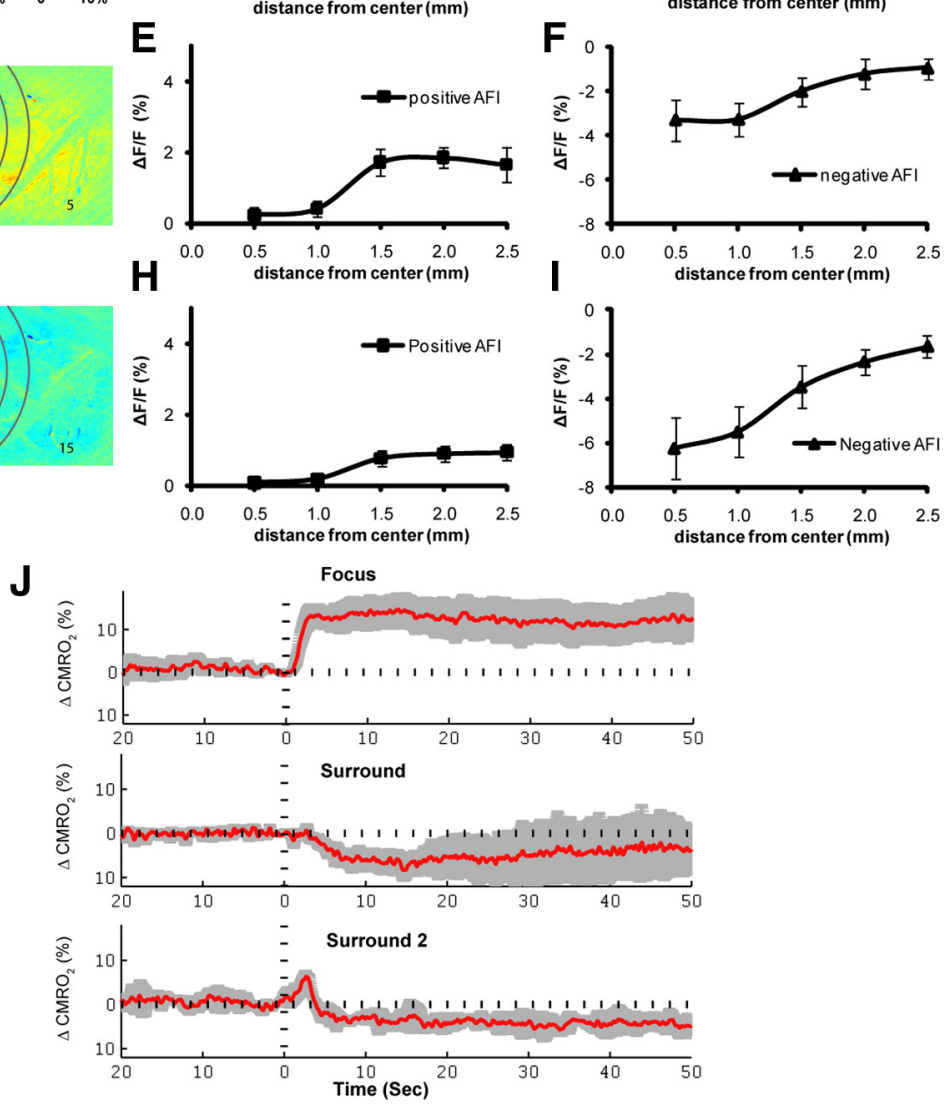

Figure 2. Cellular and tissue metabolic changes in the ictal focus and surround. $A$, AFl at selected time points after seizure onset in a single animal reveal biphasic signal with a center-surround effect during the early light phase. $\boldsymbol{B}$, The average amplitude of the positive signal during the first $2 \mathrm{~s}$ after seizure onset shows an increase in oxidative metabolism in the focus. C, Average amplitude of the negative signal shows a small decrease in oxidative metabolism in the surround. D, AFl after seizure onset at early dark phase. $\boldsymbol{E}$, Between 2 and $5 \mathrm{~s}$ after seizure onset, there is an inversion in the signal with an increase in fluorescence in the surround. $\boldsymbol{F}$, The center-surround effect is seen at this time period also showing a decrease in fluorescence in the focus. G, AFl after seizure onset at the later dark phase. $\boldsymbol{H}, \boldsymbol{I}$, As the seizure progresses $(5-40 \mathrm{~s})$, the decrease in fluorescence in the focus increases in amplitude $(\boldsymbol{H})$ and the increase in the surround (I) diminishes $\left(\boldsymbol{B}-\boldsymbol{C}, \boldsymbol{E}-\boldsymbol{F}, \boldsymbol{H}-\boldsymbol{I}: n=5\right.$ rats, 14 seizures). $\boldsymbol{J}, \mathrm{CMRO}_{2}$ increases in the focus during the ictal discharge (top, $n=9$ rats, 54 seizures). Two different responses were recorded in the surround. In most animals (middle, $n=$ 5 rats, 43 seizures), a decrease in $\mathrm{CMRO}_{2}$ was measured, while in other animals (bottom, $n=3$ rats, 39 seizures) a transient increased was followed by a longer decrease in $\mathrm{CMRO}_{2}$. Error bars, $\mathrm{SEM}$.

signal in the surround and its timing with respect to the seizure onset was then investigated in further detail. Of note, using intrinsic signal imaging in this acute seizure model, we did not find any preictal increase or decrease in either light reflection or CBV during a $20 \mathrm{~s}$ window before seizure onset.

\section{Metabolic mapping of center-surround dynamics}

In a previous article using LDF, we showed that the decrease in CBV surrounding the 4-AP focus was accompanied by a transient decrease in CBF, followed by a later increase in CBF as the seizure propagated horizontally (Zhao et al., 2009). Tissue oxygenation in the surround, on the other hand, increased throughout the duration of the seizure (Zhao et al., 2009). Although we assumed that a drop in blood flow and an increase in tissue oxygenation would be associated with a decrease in oxygen metabolism from neuronal inhibition, having not directly measured metabolism, the possibility remained that the increase in activity in inhibitory interneurons would result in an increase in metabolism, or that only a fraction of the pyramidal cells would be inhibited while others would increase their activity, resulting in a net increase or no change in metabolism. To address this question, we first investigated metabolism using AFI, so as to sample large areas of the cortex simultaneously including both the seizure focus and the surround $(n=5$ rats). With this technique, we could also determine whether the resulting maps of seizure onset were more focal than the hemodynamic maps, since the signal arises purely from local mitochondria rather than nonlocal vascular supply (Chance et al., 1979; Shibuki et al., 2003; Husson et al., 2007; Reinert et al., 2007).

AFI measured changes in the redox state of mitochondrial flavoproteins, primarily flavin adenine dinucleotide (FAD), since its oxidized form (FAD + ) is more fluorescent than the reduced form FADH (Chance et al., 1979). Increases in neuronal activity result in increases in intracellular $\mathrm{Ca}^{+}$and depletion of ATP and production of ADP, which leads to a reduction in the proton gradient across the inner mitochondrial membrane and an increase in flavoprotein fluorescence (Reinert et al., 2007; Llano et al., 2009). Similar to AFI of normal sensory processing, we found that the signal was biphasic, having an early oxidation phase (i.e., "light phase") (Fig. 2A), arising from neuronal oxidative metabolism, followed by a later, more prolonged reduction phase (i.e., "dark phase") (Fig. 2D, G), presumably arising from a combination of glycolysis in astrocytes and contamination from increased CBV and the intrinsic signal (Kasischke et al., 2004; Sirotin and Das, 2010). However, certain aspects of the AFI maps showed that epileptic events differ from normal physiologic sensory responses. In the early light phase $(0 \sim 2 \mathrm{~s})$, the maximal positive AFI peak occurred in the focus with an amplitude of $1.5 \pm 0.3 \%$ and decreased toward the periphery (ANOVA, $p<0.001$; Fig. $2 B$ ). Unlike normal physiologic responses to sensory stimulation in the neocortex, we identified an inhibited surround with a peak negative AFI of $-0.9 \pm 0.3 \%$ (ANOVA, $p<0.01$; Fig. $2 C$ ). The strong positive AFI signals were centered between 0 and $1.0 \mathrm{~mm}$, while the smaller negative AFI signals were between 1.5 and 2.5 $\mathrm{mm}$. As the seizure progressed, the signal inverted and a decrease in AFI of $-3.3 \pm 0.9 \%$ was recorded in the focus and a peak increase of $1.9 \pm 0.3 \%$ (ANOVA, $p<0.001$ ) was recorded in the surround (Fig. $2 E, F$ ). In the late part of the dark phase $(>5 \mathrm{~s})$, the negative signal in the focus reached $-6.2 \pm$ $1.4 \%$ while the positive peak in the surround decreased to a maximum of $0.9 \pm 0.2 \%$ (ANOVA, $p<0.001$; Fig. $2 H, I$ ). 
A

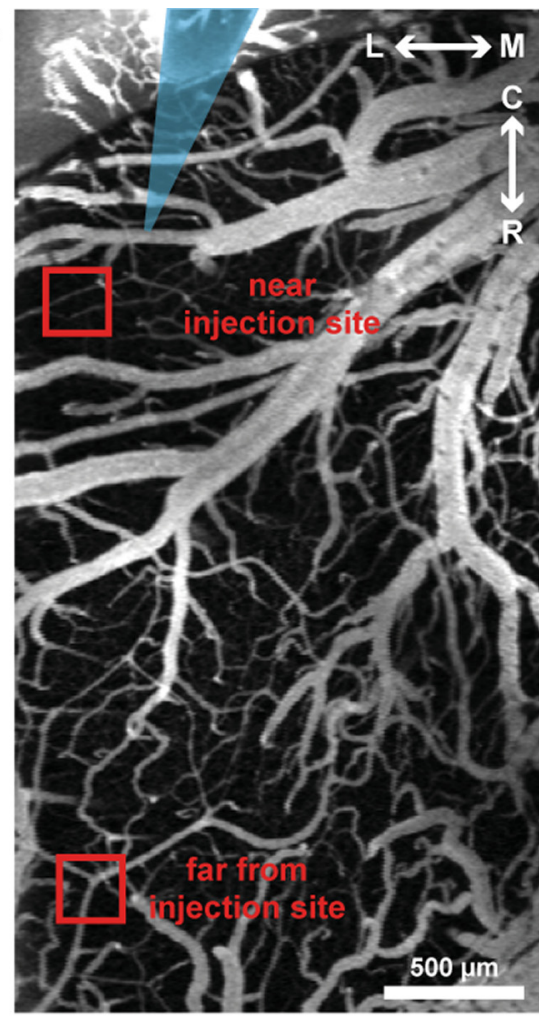

$\mathbf{F}$
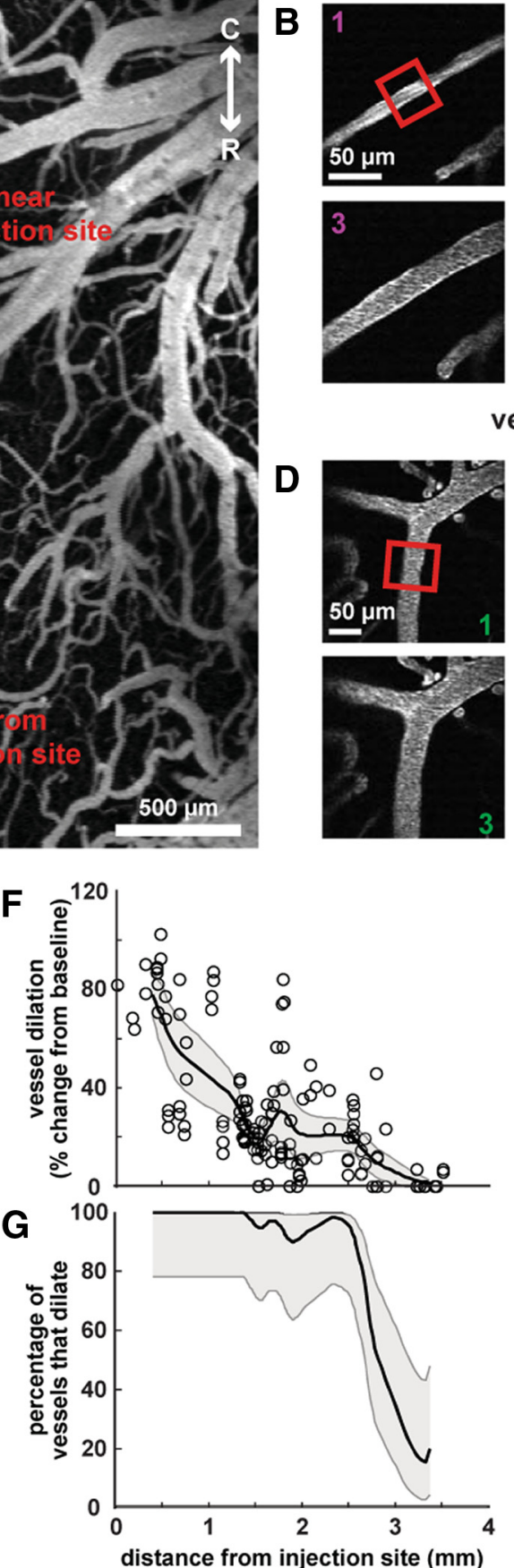

vessel near injection site $(324 \mu \mathrm{m})$
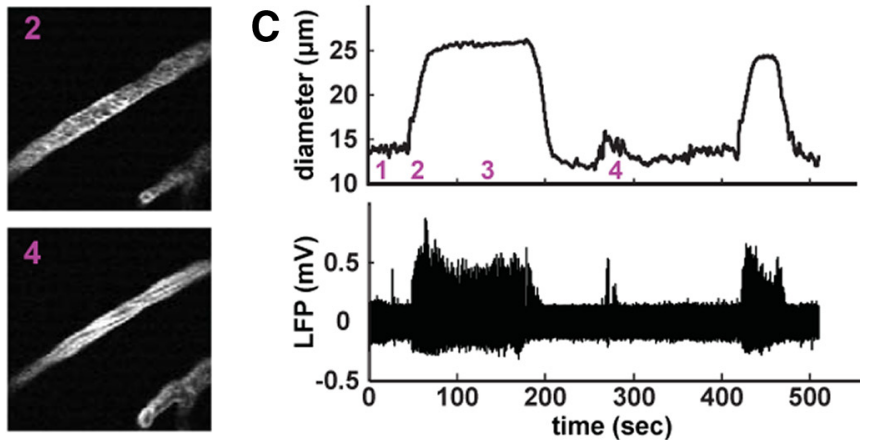

vessel far from injection site $(2160 \mu \mathrm{m})$

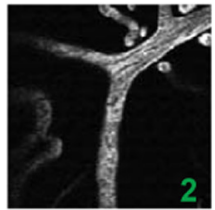

E
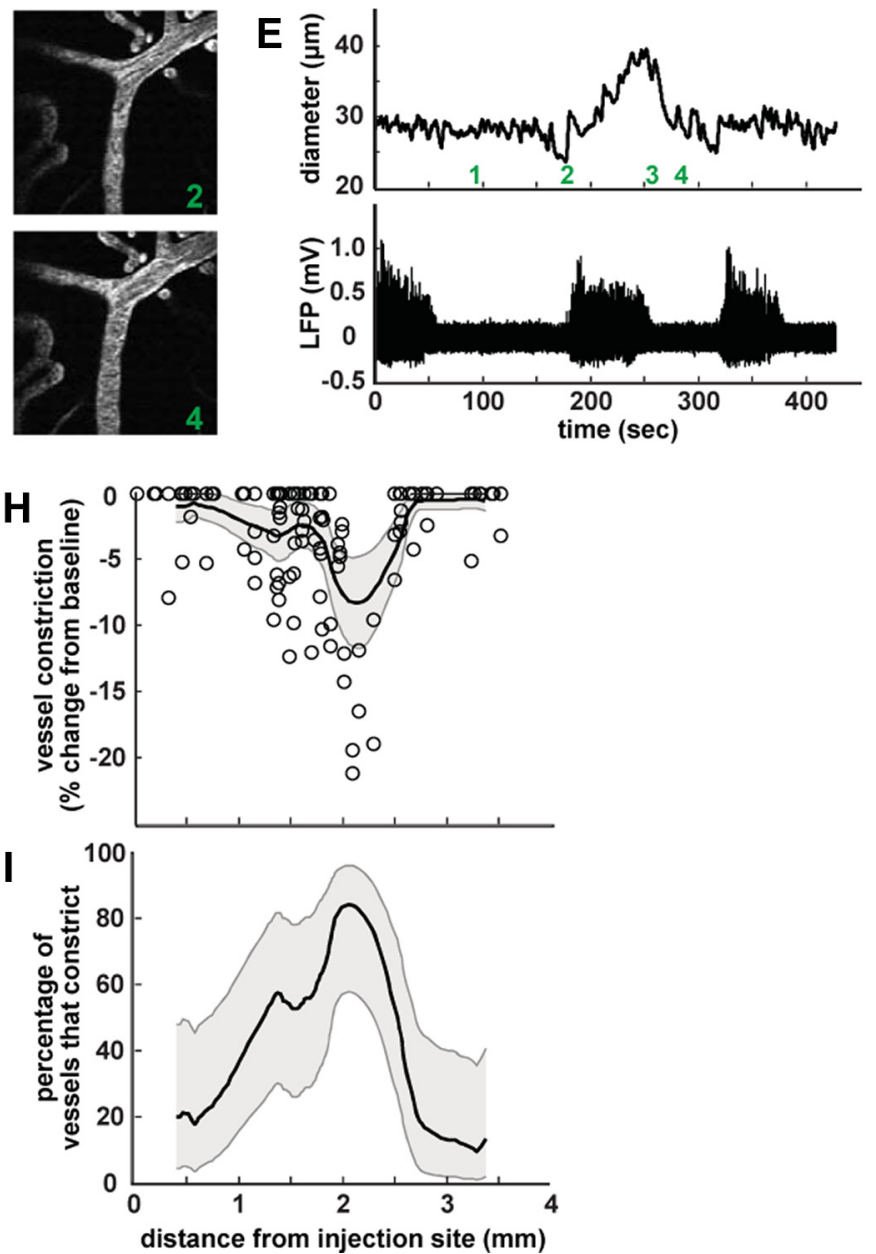

Figure 3. Seizures induce spatially dependent vascular changes. $\boldsymbol{A}$, Two-photon image of fluorescently labeled surface vasculature (blue arrow, implanted electrode; boxed areas, near and far regions from the seizure focus highlighted in parts $\boldsymbol{B}-\boldsymbol{E}) . \mathrm{L} \leftrightarrow \mathrm{M}$, Lateral-medial axis; $(\leftrightarrow R$, caudal-rostral axis. Scale bar, $500 \mu \mathrm{m}$. $\boldsymbol{B}$, Example of a representative vessel adjacent to the injection site in the focus demonstrates vascular dilation concurrent with seizure onset and evolution. $\boldsymbol{C}$, Plot of vessel diameter (above) in the red box in $\boldsymbol{B}$ during ictal events (below). Note vascular dilation with each event. Numbered timestamps in $\mathbf{C}$ top correspond to images in $\boldsymbol{B}$. D. Two-photon images in located vessel $>2 \mathrm{~mm}$ from the injection site reveals a transient constriction of arterioles at the onset of the seizure. $\boldsymbol{E}$, Plot of vessel diameter (above) in the red box in $\boldsymbol{D}$ during ictal events (below). Note vascular constriction at the onset of each event, followed by dilation. Numbered timestamps in $\boldsymbol{E}$, top, correspond to images in $\boldsymbol{D} . \boldsymbol{F}, \boldsymbol{H}$, Magnitude of maximum vessel dilation $(\boldsymbol{F})$ and vessel constriction $(\boldsymbol{H})$ as a function of distance from the focus. The shaded region represents the $95 \%$ confidence interval about the running mean is indicated by the black line. Note vascular constriction peaks $\sim 2 \mathrm{~mm}$ from the focus. $\mathbf{G}, \boldsymbol{H}$, Percentage of vessels that dilate $(\boldsymbol{G})$ and constrict $(\boldsymbol{H})$ as a function of distance from injection site, respectively. Shaded regions represent the $95 \%$ binomial confidence intervals about the percentage trend line. Note that the vast majority of measured vessels in the surround exhibit constriction.

These findings confirm an early increase in metabolism in the epileptic focus, consistent with the dip in hemoglobin and tissue oxygenation, or "initial dip," previously demonstrated in this model. However, the decrease in AFI during the early light phase surrounding the ictal focus is consistent with a transient decrease in oxidative metabolism in the surround, a newly described phenomenon that is consistent with a decrease in neuronal activity. At later time points, interpretation of the etiology of the AFI signal is less clear, resulting from a combination of changes in $\mathrm{CBV}$, which make up a significant 
proportion of the optical signal at the emission wavelength of AFI, as well as glycolysis in astrocytes, which is the primary component of the later dark phase of the AFI signal.

To overcome these temporal limitations of AFI, namely, overlap between the emission spectrum of AFI and the intrinsic CBV signal at time points $>2 \mathrm{~s}$, we directly derived tissue oxygen metabolism using simultaneous measurements of CBF and tissue oxygenation in the focus ( $n=9$ rats) and the surround $(n=8$ rats), and calculated $\mathrm{CMRO}_{2}$ using the Gjedde (2006) method (Thomsen et al., 2009). In the focus, $\mathrm{CMRO}_{2}$ was significantly increased $2.2 \mathrm{~s}$ after seizure onset (ANOVA, $p<0.05$ ) and reached a maximum increase of $14.7 \pm 3.8 \%$ (ANOVA, $p<$ $0.001)$ compared with the preictal baseline $(n=9$ rats, 54 seizures; Fig. 2J, top). In the surround region, two different phenomena were identified. In most of the rats, $\mathrm{CMRO}_{2}$ showed a significant sustained decrease by an average of $-8.3 \pm 2.5 \%$ ( 5 of 8 rats; $n=43$ seizures; ANOVA, $p<0.05)$. In the rest of the animals, we found a transient increase of $6.3 \pm 1.3 \%$ ( 1.3 to $3.2 \mathrm{~s}$; ANOVA, $p<0.05$; Fig. $2 J$, middle) followed by a sustained decrease to $-5.7 \pm 1.4 \%$ ( 3 of 8 rats; $n=39$ seizures; ANOVA, $p<$ 0.05; Fig. 2 J, bottom). These results show the expected dramatic increase in metabolism in the focus and confirm the AFI results showing an overall decrease in metabolism in the surround consistent with a net decrease in neuronal activity. However, unlike the AFI results, the measurements of tissue metabolism persist for a longer time period, supporting the correlation between only the early light phase of the AFI data and neuronal metabolism.

\section{Two-photon measurements of arteriolar diameter}

To determine the etiology of the transient drop in CBF and CBV in the surround at seizure onset, we measured arteriolar diameter using two-photon imaging ( $n=4$ rats). Low-magnification images were first used to navigate the surface vasculature and determine spatial distances of specific vessels relative to the injection site of the 4-AP (Fig. 3A). High-magnification movies of individual arterioles allowed for tracking diameter changes during seizure activity near (Fig. $3 B, C$ ) and far (Fig. $3 D, E$ ) from the seizure focus. We found that arterioles dilated in response to the seizure, with a decreasing amount of dilation with increasing distance from the 4-AP injection site (Fig. $3 F)(n=4$ rats, 71 vessels, 45 seizures, 143 measurements). Ninety-seven percent of the measured arterioles within $2.5 \mathrm{~mm}$ of the seizure focus dilated (Fig. $3 G$ ), with little response for vessels further away. For vessels in a 1 $\mathrm{mm}$ ring centered on the 4-AP injection site (focus), arterioles dilated by an average of $63 \pm 5 \%$ of their baseline diameter (Fig. $3 F$ ). In the ring $1.5-2.5 \mathrm{~mm}$ from the 4-AP injection site (surround), we observed early vascular constriction followed by delayed dilation in all seizures measured (Fig. $3 E, H$ ). In this ring, $69 \%$ of the vessels displayed this early constriction, with a diminished fraction of vessels dilating for vessels closer or farther from the seizure focus (Fig. 3I). On average, the vessels in this ring constricted by $7 \pm 1 \%$ of their baseline diameter during this constriction phase, with smaller constrictions for vessels closer or farther from the seizure focus (Fig. $3 H$ ).

\section{Temporal characteristics of vascular reactivity}

Having demonstrated for the first time that active vascular constriction in the ictal surround occurs, we sought to determine the timing of this event with respect to seizure onset. Plotting the temporal profile of vasodilation compared with vasoconstriction (Fig. $4 A, B$ ), we determined that vasodilation in the focus occurred $0.5 \pm 0.1 \mathrm{~s}$ after seizure onset, whereas vasoconstriction in
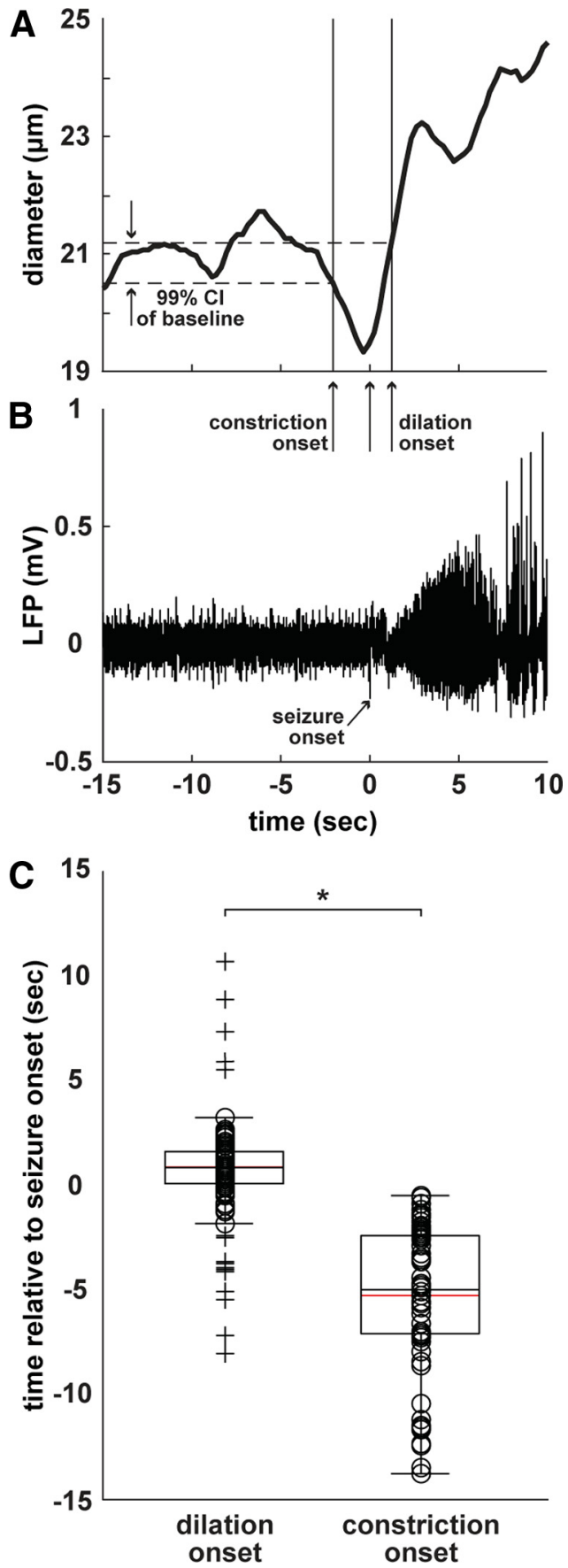

Figure 4. Arterioles in the surround constrict before seizure onset. $\boldsymbol{A}, \boldsymbol{B}$, Representative example of vascular diameter $(\boldsymbol{A})$ and simultaneous LFP recording of seizure onset $(\boldsymbol{B})$ demonstrate preictal vasoconstriction and postictal vasodilation. A $99 \%$ confidence interval about the mean diameter is shown in $\boldsymbol{A}$. Arrows indicate constriction (first arrow), seizure onset (second arrow), and dilation (third arrow). C, Boxplot of dilation and constriction onset times relative to seizure onset. Red and black lines represent mean and median respectively. Circles are individual data points, and cross-hairs are statistical outliers. ${ }^{*} p<1.0 \times 10^{-7}$ ( $n=4$ rats, 71 vessels, 45 total seizures, 143 total measurements).

the surround occurred $5.3 \pm 0.5 \mathrm{~s}$ before seizure onset (Fig. $4 C$ ) $\left(p<1.0 \times 10^{-7}\right.$, Mann-Whitney $U$ test). Note that all vasoconstriction was observed to occur before seizure onset. There was no significant correlation between either the onset time of constriction or dilation and the seizure duration, arteriole diameter, and maximum LFP amplitude (Fig. 5). 


\section{Discussion}

Our data indicate that, in a model of acute rodent seizures, ictal onset is preceded by active vasoconstriction in small arterioles surrounding the ictal focus. As the seizure progresses, tissue and oxidative metabolism increase in the ictal focus and generally decrease in the surround, as do CBV and cell swelling, although transient increases in tissue metabolism can occur in the surround in some seizures. Our data support a model in which a centersurround dynamic characterizes both metabolic and neurovascular mechanisms during in vivo ictal evolution. In addition, the novel preictal events we observed may be a useful tool for seizure prediction and are possible to be manipulated or capitalized upon for therapeutic effect.
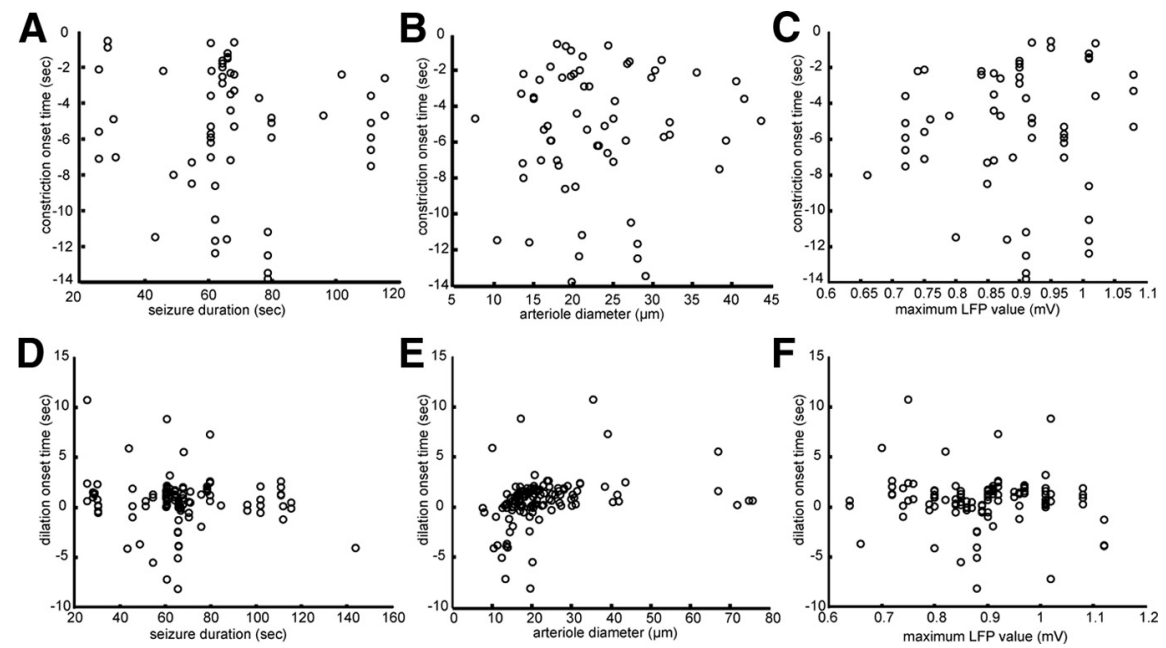

Figure 5. $\boldsymbol{A}-\boldsymbol{F}$, Constriction and dilation onset time as a function of seizure duration $(\boldsymbol{A}, \boldsymbol{D})$, arteriole diameter $(\boldsymbol{B}, \boldsymbol{E})$, and maximum LFP value $(\boldsymbol{C}, \boldsymbol{F})$. The plots indicate that both onset times show a poor correlation with the investigated parameters. Each circle represents an individual data point.

\section{Center-surround phenomena}

Epileptic surround inhibition elicited by interictal spikes was thought to be a mechanism to prevent the interictal-to-ictal transition (Prince and Wilder, 1967). Similar surround inhibition has been described during normal cortical processing (Derdikman et al., 2003; Devor et al., 2007) as well as during interictal spike activity (Schwartz and Bonhoeffer, 2001). This finding may be unique to the neocortex and is not found in the cerebellum where perforant pathway stimulation elicits upstream vasodilation of larger arterioles and a nonspecific widespread increase in $\mathrm{CBF}$ (Iadecola et al., 1997). Ictal events are more complicated. The concept of surround inhibition has not been well studied during seizures in vivo, although evidence of enhanced inhibitory activity around the ictal focus is mentioned (Bragin et al., 2002). Ictal surround inhibition does not appear to completely encircle the focus, which is consistent with the center-surround phenomenon reported during normal cortical activity (Devor et al., 2007). Explanations for such an inhomogeneous response include a mixture of inhibitory and excitatory neuronal activity or a reflection of patchy vascular domains. In this study, we show clear evidence of early ictal surround inhibition, revealed with optical mapping of light scattering from cell swelling and CBV, which is further manifested in tissue and cellular metabolism and preictal arteriolar vasoreactivity.

Associated with a decrease in neuronal activity in the ictal surround, we demonstrate a decrease in CBV. The etiology of the widespread decrease in $\mathrm{CBV}$ in the surround was hypothesized to arise either from a passive steal phenomenon, as vessels in the focus dilate and redirect oxygenated blood toward the metabolically hyperactive focus (Hirase et al., 2004a), or from active shunting of blood through vasoconstriction of vessels in the surround (Zhao et al., 2009). This latter mechanism has been demonstrated to occur during normal cortical processing. However, surround vasoconstriction during normal cortical processing occurred after physiologic stimulation and after a brief period of vasodilation (Devor et al., 2007). In contrast, we report the opposite time profile of dilation and constriction in the surround of an epileptic seizure, namely, preictal vasoconstriction followed by vasodilation. Surprisingly, the spatial topography, if not the timing, of the center-surround inversion in vascular reactivity is conserved. Studies of the blood oxygen level-dependent signals during normal visual processing in the monkey also show inversion at $>2 \mathrm{~mm}$ (Shmuel et al., 2006), similar to what we have shown for ictal events using two-photon imaging. The conservation of the center-surround effect and its spatial topography in both normal and abnormal physiologic events points to a conserved mechanism. Recent evidence is mounting that vascular reactivity at the arteriolar level is highly influenced by local neurotransmitter and neuropeptide release (Hamel, 2006), local potassium (Filosa et al., 2006), and astrocyte- and pericyte-mediated signaling (Peppiatt et al., 2006; Wang et al., 2006).

\section{Preictal surround vasoconstriction}

Preictal increases and decreases in neuronal activity, and progressive coalescing of high-frequency activity and microseizures have been documented (Litt et al., 2001). It is likely that these preictal electrophysiological events are accompanied by alterations in focal hemodynamic responses. However, no preictal decreases in high-frequency activity in the surround have been demonstrated, although human single-unit recordings have shown that a small percentage of neurons may decrease activity during the immediate preictal activity (Truccolo et al., 2011). Indeed, preictal vascular reactivity was first theorized (mistakenly at the time) to be the etiology for seizure activity as early as 1933 (Gibbs, 1933). More recent studies have demonstrated increases in lobar perfusion as early as $20 \mathrm{~min}$ before focal as well as generalized spikeand-wave events (Diehl et al., 1998). Likewise, both increases (Mäkiranta et al., 2005) and decreases (Hoshi and Tamura, 1992) in tissue oxygenation have been found tens of seconds before seizure onset using fMRI and near-infrared spectroscopy. IOS imaging has also demonstrated focal preictal changes in light reflection in animal models, and increases in CBV and decreases in hemoglobin oxygenation in human epileptic cortex almost $20 \mathrm{~s}$ before seizure onset (Zhao et al., 2007). However, this is the first report of active preictal vasoconstriction surrounding an ictal focus. Another report of two-photon imaging of blood flow in and around an epileptic focus used an interictal model (Hirase et al., 2004b). Our data are consistent with a model of preictal vasoconstriction in the cortex surrounding the ictal focus as crucial for initiating seizure onset. Since we did not perform electrical recordings from the surround, we cannot say whether this preictal vascular activity correlates with early decreases in neuronal activity. 
Another hypothesis rests on the concept of anticipatory shunting of oxygenated blood to the ictal focus, possibly precipitated by a gradual build-up of excitatory activity. A similar anticipatory increase in CBV has been demonstrated before normal cortical processing in visual cortex (Sirotin and Das, 2009). However, if the pial arterioles we are imaging around the focus provide blood flow to the focus itself, we would expect a decrease in flow in the focus, which we do not find. Alternatively, the pial arterioles in the surround may only control very local blood flow and have no influence on blood flow in the focus. This would imply that there is a transient decrease in metabolism in the surround before the seizure begins followed by an increase in metabolism in the focus. However, we did not find any preictal changes in metabolism using AFI and direct tissue measurements, although slight metabolic changes may be below the resolution of our techniques. Finally, there is the more controversial and less likely possibility that the hemodynamic events are primary and the electrographic events secondary. This hypothesis has been raised for both epileptic activity (Hawco et al., 2007) and normal cortical processing (Moore and Cao, 2008). Along this line of argument, preictal vasoconstriction would shunt oxygenated blood to the focus, which would then facilitate or directly cause ictal onset.

\section{Ictal metabolism in the focus and surround}

Despite the discovery of preictal surround vasoconstriction, we did not find any evidence of significant preictal alterations in metabolism in either the focus or the surround. Not surprisingly, a clear increase in metabolism was found in the focus. The sustained increase in metabolism in the ictal focus is consistent with prior work using either autoradiography or PET. Although human PET studies show interictal hypometabolism (Franck et al., 1986), intense interictal activity causes focal hypermetabolism with surround hypometabolism (Bruehl and Witte, 1995). Ictal events, on the other hand, always show increases in glucose and oxygen metabolism in the focus (Engel et al., 1982). Although AFI imaging has been used extensively to map normal sensory processing (Shibuki et al., 2003; Husson et al., 2007; Sirotin and Das, 2010), only one prior report exists of AFI imaging of epilepsy (Takao et al., 2006). In this article, we show for the first time that AFI signals are exquisitely sensitive to the ictal onset during the light phase of imaging as early as $0-2 \mathrm{~s}$ after ictal onset. Hence, AFI imaging may be a useful technique for localizing epileptic seizures, possibly providing a map for therapeutic intervention that may be more focal than CBV or hemoglobin oxygenation maps derived from the IOS.

Metabolism in the surround of an ictal focus has not been well studied. Our results provide direct evidence of a drop in tissue metabolism surrounding the ictal focus, which is consistent with the existence of neuronal inhibition in these areas and consistent with in vivo models of "feed-forward" or "veto" inhibition shaping the epileptic wavefront as it propagates (Trevelyan et al., 2007). Decreased AFI fluorescence in areas of cortex surrounding areas of focal activation has been correlated with neuronal inhibition in the cerebellum (Reinert et al., 2007). However, AFI imaging of the surround metabolism has not yet been reported in the neocortex. In summary, we have identified a novel preictal vascular phenomenon, namely, surround vasoconstriction. Although the precise etiology of this event is unclear, a transient rise in preictal inhibition, which serves to increase synchronization, is a possible candidate. We have also shown that AFI imaging, particularly during the early light phase, is useful at mapping the ictal onset zone, and its clinical applications should be explored, as has been done with optical imaging of intrinsic signals (Zhao et al.,
2007). Finally, we find support for a center-surround dynamic at play in ictal propagation, lending further support for surround inhibition in shaping the evolving ictal wavefront.

\section{References}

Bragin A, Mody I, Wilson CL, Engel J Jr (2002) Local generation of fast ripples in epileptic brain. J Neurosci 22:2012-2021.

Bruehl C, Witte OW (1995) Cellular activity underlying altered brain metabolism during focal epileptic activity. Ann Neurol 38:414-420.

Chance B, Schoener B, Oshino R, Itshak F, Nakase Y (1979) Oxidationreduction ratio studies of mitochondria in freeze-trapped samples. NADH and flavoprotein fluorescence signals. J Biol Chem 254:47644771.

Derdikman D, Hildesheim R, Ahissar E, Arieli A, Grinvald A (2003) Imaging spatiotemporal dynamics of surround inhibition in the barrels somatosensory cortex. J Neurosci 23:3100-3105.

Devor A, Tian P, Nishimura N, Teng IC, Hillman EM, Narayanan SN, Ulbert I, Boas DA, Kleinfeld D, Dale AM (2007) Suppressed neuronal activity and concurrent arteriolar vasoconstriction may explain negative blood oxygenation level-dependent signal. J Neurosci 27:4452-4459.

Diehl B, Knecht S, Deppe M, Young C, Stodieck SR (1998) Cerebral hemodynamic response to generalized spike-wave discharges. Epilepsia 39: $1284-1289$.

Engel J Jr, Kuhl DE, Phelps ME (1982) Patterns of human local cerebral glucose metabolism during epileptic seizures. Science 218:64-66.

Filosa JA, Bonev AD, Straub SV, Meredith AL, Wilkerson MK, Aldrich RW, Nelson MT (2006) Local potassium signaling couples neuronal activity to vasodilation in the brain. Nat Neurosci 9:1397-1403.

Folbergrová J, Ingvar M, Siesjö BK (1981) Metabolic changes in cerebral cortex, hippocampus, and cerebellum during sustained bicucullineinduced seizures. J Neurochem 37:1228-1238.

Franck G, Sadzot B, Salmon E, Depresseux JC, Grisar T, Peters JM, Guillaume M, Quaglia L, Delfiore G, Lamotte D (1986) Regional cerebral blood flow and metabolic rates in human focal epilepsy and status epilepticus. Adv Neurol 44:935-948.

Gibbs FA (1933) Cerebral blood flow preceding and accompanying experimental convulsions Arch Neurol Psychiat Chicago 30:1003-1010.

Gjedde A (2006) Blood-Brain Transfer and Metabolism of Oxygen. In: Blood-brain barriers: from ontogeny to artificial interfaces (Dermietzel $\mathrm{R}$, Spray DC, Nedergaard M, eds), pp 2 v. (xxxii, 741 p.). Weinheim: Wiley-VCH.

Hamel E (2006) Perivascular nerves and the regulation of cerebrovascular tone. J Appl Physiol 100:1059-1064.

Hawco CS, Bagshaw AP, Lu Y, Dubeau F, Gotman J (2007) BOLD changes occur prior to epileptic spikes seen on scalp EEG. Neuroimage 35:1450-1458.

Hirase H, Creso J, Buzsáki G (2004a) Capillary level imaging of local cerebral blood flow in bicuculline-induced epileptic foci. Neuroscience 128:209-216.

Hirase H, Qian L, Barthó P, Buzsáki G (2004b) Calcium dynamics of cortical astrocytic networks in vivo. PLoS Biol 2:E96.

Hoshi Y, Tamura M (1992) Cerebral oxygenation state in chemicallyinduced seizures in the rat-study by near infrared spectrophotometry. Adv Exp Med Biol 316:137-142.

Husson TR, Mallik AK, Zhang JX, Issa NP (2007) Functional imaging of primary visual cortex using flavoprotein autofluorescence. J Neurosci 27:8665-8675.

Iadecola C, Nedergaard M (2007) Glial regulation of the cerebral microvasculature. Nat Neurosci 10:1369-1376.

Iadecola C, Yang G, Ebner TJ, Chen G (1997) Local and propagated vascular responses evoked by focal synaptic activity in cerebellar cortex. J Neurophysiol 78:651-659.

Ingvar M (1986) Cerebral blood flow and metabolic rate during seizures. Relationship to epileptic brain damage. Ann N Y Acad Sci 462:194-206.

Kasischke KA, Vishwasrao HD, Fisher PJ, Zipfel WR, Webb WW (2004) Neural activity triggers neuronal oxidative metabolism followed by astrocytic glycolysis. Science 305:99-103.

Kobayashi E, Hawco CS, Grova C, Dubeau F, Gotman J (2006) Widespread and intense BOLD changes during brief focal electrographic seizures. Neurology 66:1049-1055.

Kreisman NR, Magee JC, Brizzee BL (1991) Relative hypoperfusion in rat 
cerebral cortex during recurrent seizures. J Cereb Blood Flow Metab 11:77-87.

Litt B, Esteller R, Echauz J, D’Alessandro M, Shor R, Henry T, Pennell P, Epstein C, Bakay R, Dichter M, Vachtsevanos G (2001) Epileptic seizures may begin hours in advance of clinical onset: a report of five patients. Neuron 30:51-64.

Llano DA, Theyel BB, Mallik AK, Sherman SM, Issa NP (2009) Rapid and sensitive mapping of long-range connections in vitro using flavoprotein autofluorescence imaging combined with laser photostimulation. J Neurophysiol 101:3325-3340.

Mäkiranta M, Ruohonen J, Suominen K, Niinimäki J, Sonkajärvi E, Kiviniemi V, Seppänen T, Alahuhta S, Jäntti V, Tervonen O (2005) BOLD signal increase preceeds EEG spike activity-a dynamic penicillin induced focal epilepsy in deep anesthesia. Neuroimage 27:715-724.

Masamoto K, Omura T, Takizawa N, Kobayashi H, Katura T, Maki A, Kawaguchi H, Tanishita K (2003) Biphasic changes in tissue partial pressure of oxygen closely related to localized neural activity in guinea pig auditory cortex. J Cereb Blood Flow Metab 23:1075-1084.

Moore CI, Cao R (2008) The hemo-neural hypothesis: on the role of blood flow in information processing. J Neurophysiol 99:2035-2047.

Motamedi GK, Salazar P, Smith EL, Lesser RP, Webber WR, Ortinski PI, Vicini S, Rogawski MA (2006) Termination of epileptiform activity by cooling in rat hippocampal slice epilepsy models. Epilepsy Res 70:200210.

Nguyen QT, Tsai PS, Kleinfeld D (2006) MPScope: a versatile software suite for multiphoton microscopy. J Neurosci Methods 156:351-359.

Offenhauser N, Thomsen K, Caesar K, Lauritzen M (2005) Activity-induced tissue oxygenation changes in rat cerebellar cortex: interplay of postsynaptic activation and blood flow. J Physiol 565:279-294.

Peppiatt CM, Howarth C, Mobbs P, Attwell D (2006) Bidirectional control of CNS capillary diameter by pericytes. Nature 443:700-704.

Prince DA, Wilder BJ (1967) Control mechanisms in cortical epileptogenic foci. "Surround" inhibition. Arch Neurol 16:194-202.

Raichle ME, Mintun MA (2006) Brain work and brain imaging. Annu Rev Neurosci 29:449-476.

Reinert KC, Gao W, Chen G, Ebner TJ (2007) Flavoprotein autofluorescence imaging in the cerebellar cortex in vivo. J Neurosci Res 85:3221-3232.

Rensing N, Ouyang Y, Yang XF, Yamada KA, Rothman SM, Wong M (2005) In vivo imaging of dendritic spines during electrographic seizures. Ann Neurol 58:888-898.

Roche-Labarbe N, Zaaimi B, Berquin P, Nehlig A, Grebe R, Wallois F (2008) NIRS-measured oxy- and deoxyhemoglobin changes associated with EEG spike-and-wave discharges in children. Epilepsia 49:1871-1880.

Salzberg BM, Obaid AL, Gainer H (1985) Large and rapid changes in light scattering accompany secretion by nerve terminals in the mammalian neurohypophysis. J Gen Physiol 86:395-411.
Schwartz TH, Bonhoeffer T (2001) In vivo optical mapping of epileptic foci and surround inhibition in ferret cerebral cortex. Nat Med 7:1063-1067.

Shibuki K, Hishida R, Murakami H, Kudoh M, Kawaguchi T, Watanabe M, Watanabe S, Kouuchi T, Tanaka R (2003) Dynamic imaging of somatosensory cortical activity in the rat visualized by flavoprotein autofluorescence. J Physiol 549:919-927.

Shmuel A, Augath M, Oeltermann A, Logothetis NK (2006) Negative functional MRI response correlates with decreases in neuronal activity in monkey visual area V1. Nat Neurosci 9:569-577.

Sirotin YB, Das A (2009) Anticipatory haemodynamic signals in sensory cortex not predicted by local neuronal activity. Nature 457:475-479.

Sirotin YB, Das A (2010) Spatial Relationship between Flavoprotein Fluorescence and the Hemodynamic Response in the Primary Visual Cortex of Alert Macaque Monkeys. Front Neuroenergetics 2:6.

Skarphedinsson JO, Hårding H, Thorén P (1988) Repeated measurements of cerebral blood flow in rats. Comparison between hydrogen clearance method and laser Doppler Flowmetry. Acta Physiol Scand 134:133-142.

Takao T, Murakami H, Fukuda M, Kawaguchi T, Kakita A, Takahashi H, Kudoh M, Tanaka R, Shibuki K (2006) Transcranial imaging of audiogenic epileptic foci in the cortex of DBA/2J mice. Neuroreport 17: 267-271.

Thomsen K, Piilgaard H, Gjedde A, Bonvento G, Lauritzen M (2009) Principal cell spiking, postsynaptic excitation, and oxygen consumption in the rat cerebellar cortex. J Neurophysiol 102:1503-1512.

Trevelyan AJ, Sussillo D, Yuste R (2007) Feedforward inhibition contributes to the control of epileptiform propagation speed. J Neurosci 27:3383-3387.

Truccolo W, Donoghue JA, Hochberg LR, Eskandar EN, Madsen JR, Anderson WS, Brown EN, Halgren E, Cash SS (2011) Single-neuron dynamics in human focal epilepsy. Nat Neurosci 14:635-641.

Wang X, Lou N, Xu Q, Tian GF, Peng WG, Han X, Kang J, Takano T, Nedergaard M (2006) Astrocytic Ca2+ signaling evoked by sensory stimulation in vivo. Nat Neurosci 9:816-823.

Xu L, Rensing N, Yang XF, Zhang HX, Thio LL, Rothman SM, Weisenfeld AE, Wong M, Yamada KA (2008) Leptin inhibits 4-aminopyridine- and pentylenetetrazole-induced seizures and AMPAR-mediated synaptic transmission in rodents. J Clin Invest 118:272-280.

Zhao M, Suh M, Ma H, Perry C, Geneslaw A, Schwartz TH (2007) Focal increases in perfusion and decreases in hemoglobin oxygenation precede seizure onset in spontaneous human epilepsy. Epilepsia 48:2059-2067.

Zhao M, Ma H, Suh M, Schwartz TH (2009) Spatiotemporal dynamics of perfusion and oximetry during ictal discharges in the rat neocortex. J Neurosci 29:2814-2823.

Zhu XH, Zhang Y, Tian RX, Lei H, Zhang N, Zhang X, Merkle H, Ugurbil K, Chen W (2002) Development of (17)O NMR approach for fast imaging of cerebral metabolic rate of oxygen in rat brain at high field. Proc Natl Acad Sci U S A 99:13194-13199. 